sistance mensuel. On a retenu uniquement les premières lactations et considéré 2 périodes : 90 premiers jours et lactation totale. Toutes les données correspondant à ces 2 périodes ont été corrigées pour l'âge au premier vêlage et transformées en probit. Ces premiers résultats concernent 50 taureaux : 53 filles en moyenne pour le lait, le TB et le TP, 36 filles pour les données calcium. L'héritabilité des quantités est assez élevée $(0,35$ à 0,49$)$. Les taux ont une $h^{2}$ inférieure ou égale aux quantités. L' $h^{3}$ du coefficient de persistance est faible $(0,13)$.

En ce qui concerne les corrélations génétiques, il faut souligner les résultats suivants : $1^{\circ}$ Il existe une liaison très étroite entre quantité de lait d'une part et quantités de graisse et de protéines d'autre part $(0,9$ à 1,0$) .2^{\circ}$ La quantité de lait est en corrélation négative avec le taux de protéines $(-0,28)$ mais surtout avec le taux butyreux $(-0,41) .3^{\circ}$ Le rapport protéines /graisse des laits est en corrélation positive avec la quantité de lait $(+0,22)$ et avec la quantité de protéines $(+0,31)$ mais en corrélation nulle avec la quantité de matière grasse. Seule une sélection sur la quantité de protéines peut améliorer le rapport protéines /graisse des laits qui conditionne en partie leur valeur fromagère (La corrélation génétique entre le rapport TP/TB et le TP est de $+0,40$; elle est de $-0,82$ entre le rapport TP /TB et le TB). $4^{\circ}$ Le coefficient de persistance est en corrélation positive avec les quantités $(0,44$ à 0,78$)$ et pratiquement indépendant des taux, sauf du taux de calcium. Il est également indépendant de la production maximum journalière. $5^{\circ}$ Les corrélations entre les 2 périodes sont respectivement de $0,82,0,72,0,82$ et 0,73 pour les quantités de lait, de matière grasse, de protéines et de calcium, de $0,87,0,86,0,90$ et 0,97 pour les TB, TP, TCa et TP/TB.

\title{
VARIATION DES PARAMÈTRES GÉNÉTIQUES DE LA PRODUCTION LAITIÈRE DES BREBIS AVEC LE NIVEAU D'ÉTABLE
}

\author{
J. Romer. - Institut de Zootechnie, Cracovie (Pologne).
}

J.-C. Flamant, J.-J. Collead, M. Poutous. - Station de Génétique quantitative et appliquée, C.N.R.Z., 78-Jouy-en-Josas.

Les performances moyennes annuelles (production laitière à la retraite) de 58 troupeaux de brebis de race Lacaune, contrôlés dans le rayon de Roquefort durant au moins 7 années successives (1960 à 1966 inclus) et représentant 65000 lactations, ont été classées et réparties en 3 groupes selon leur niveau. Le niveau " haut " et le niveau "bas " représentent respectivement le quart supérieur et le quart inférieur des moyennes classées. Pour chacun des 3 niveaux et chacun des groupes d'âges de brebis ( 1 an, 3 ans et 4 ans) ont été déterminés les coefficients d'héritabilité de la production laitière à la traite $(1402,2341$ et 1352 couples mères-filles respectivement pour les niveaux haut, moyen et bas) et les coefficients de corrélation génétique entre la production laitière à la traite et le niveau du contrôle maximum. Les valeurs suivantes ont été trouvées pour les coefficients d'héritabilité à niveau d'étable décroissant : 1 an $0,32,0,30,0,28 ; 3$ ans $0,31,0,14,0,50 ; 4$ ans 0,$23 ; 0,27 ; 0,39$. On n'observe pas de variation significative avec l'âge et avec le niveau de production. Cependant, la variance résiduelle tend à augmenter plus nettement que la variance génétique avec la moyenne. Ces résultats montrent qu'il n'est pas nécessaire de réaliser le testage des béliers dans une classe particulière bien que l'échelle de classement des différents béliers puisse être plus large dans les troupeaux de niveau de production élevé. En première lactation à $1 \mathrm{an}$, la corrélation génétique entre la production laitière à la traite et la production au contrôle maximum tend à être plus élevée dans le niveau "bas " que dans le niveau "haut ". Cependant, les valeurs trouvées ne sont pas significativement différentes de $r=1,00$. Pour chacun des 3 niveaux " haut ", "moyen " et "bas ", les valeurs suivantes ont été trouvées : 1 an 0,84 , $0,98,1,01 ; 3$ ans $0,87,0,92,0,93 ; 4$ ans $0,90,0,96,0,87$. 